\title{
Survey on Vietnamese teachers' perspectives and perceived support during COVID-19
}

\author{
Authors \\ Cam-Tu Vu, Anh-Duc Hoang, Van-Quan Than 3 , Manh-Tuan Nguyen 4 , Viet-Hung \\ Dinh $_{5}$, Quynh-Anh Thi Le, Thu-Trang Thi Le6, Hiep-Hung Pham 1,7 , Yen-Chi Nguyen 2
}

\author{
Affiliations \\ 1. Institute of Theoretical and Applied Research, Duy Tan University, Hanoi 100000, \\ Vietnam \\ 2. EdLab Asia Educational Research and Development Centre, Hanoi 10000o, Vietnam \\ 3. Political Academy, Hanoi 100000, Vietnam \\ 4. Early Childhood Department, Hanoi National University of Education, Hanoi \\ 100000, Vietnam \\ 5. University of Labour and Social Affairs, Hanoi 100000, Vietnam \\ 6. Hanoi University, Hanoi 100000, Vietnam \\ 7. Phu Xuan University, Hue 49000, Vietnam
}

\section{Corresponding author(s)}

Anh-Duc Hoang (duc@edlabasia.org)

\begin{abstract}
The COVID-19 pandemic has caused unprecedented damage to the educational system worldwide. Besides the measurable economic impacts in the short-term and long-term, there is intangible destruction within educational institutions. In particular, teachers the most critical intellectual resources of any schools - have to face various types of financial, physical, and mental struggles due to COVID-19. To capture the current context of more than one million Vietnamese teachers during COVID-19, we distributed an esurvey to more than 2,500 randomly selected teachers from two major teacher communities on Facebook from 6th to 11th April 2020. From over 373 responses, we excluded the observations which violated our cross-check questions and retained 294 observations for further analysis. This dataset includes: (i) Demographics of participants; (ii) Teachers' perspectives regarding the operation of teaching activities during the pandemic; (iii) Teachers' received support from their schools, government bodies, other stakeholders such as teacher unions, and parents' associations; and (iv) teachers' evaluation of school readiness toward digital transformation. Further, the dataset was supplemented with an additional question on the teachers' primary source of professional development activities during the pandemic.
\end{abstract}




\section{Keywords}

Education management; Teacher satisfaction; Teacher engagement; COVID-19; Vietnam

\section{Specifications Table}

\begin{tabular}{|c|c|}
\hline Subject & Education, Education Management \\
\hline $\begin{array}{l}\text { Specific subject } \\
\text { area }\end{array}$ & $\begin{array}{l}\text { Education Management; School Effectiveness; Teacher } \\
\text { Satisfaction }\end{array}$ \\
\hline Type of data & Raw data in excel file and analyzed data \\
\hline $\begin{array}{l}\text { How data were } \\
\text { acquired }\end{array}$ & $\begin{array}{l}\text { Data was gathered using an online survey and converted into } \\
\text { the .xlsx format for formal analysis in SPSS v.20. }\end{array}$ \\
\hline Data format & $\begin{array}{l}\text { Raw } \\
\text { Analyzed }\end{array}$ \\
\hline $\begin{array}{l}\text { Parameters for } \\
\text { data collection }\end{array}$ & $\begin{array}{l}\text { The target population of this work is Vietnamese teachers } \\
\text { whose teaching profession was affected by the COVID-19 } \\
\text { pandemic. In light of the national school closures policy, } \\
\text { almost every educational institution has to close until the end } \\
\text { of April } 2020 \text {. As a result, approximately one million teachers } \\
\text { of various school types and educational levels were affected. }\end{array}$ \\
\hline $\begin{array}{l}\text { Description of data } \\
\text { collection }\end{array}$ & $\begin{array}{l}\text { An online survey has been delivered to 2,500 randomly } \\
\text { selected Pre-K to post-secondary teachers. They are members } \\
\text { of two major teacher communities on Facebook: MIE Expert } \\
\text { Vietnam ( } 38,600 \text { members) and Vietnam Innovative } \\
\text { Education Forum (14,000 members). }\end{array}$ \\
\hline $\begin{array}{l}\text { Data source } \\
\text { location }\end{array}$ & $\begin{array}{l}\text { Information is collected from secondary student institutes in } \\
\text { Hanoi (Latitude } 21^{\circ} 1^{\prime} 28.2^{\prime \prime N} \text {, Longitude } 105^{\circ} 50^{\prime} 28.21^{\prime \prime} \mathrm{E} \text {, } \\
\text { Vietnam. }\end{array}$ \\
\hline Data accessibility & $\begin{array}{l}\text { Repository name: Harvard Dataverse } \\
\text { Data identification number: } \\
\text { Direct URL to data: https://doi.org/10.7910/DVN/FOCPKH, } \\
\text { Harvard Dataverse, V1 } \\
\text { Repository Name: Mendeley } \\
\text { Direct URL to data: } \\
\text { https://data.mendeley.com/datasets/cy46h2rvwg/draft?a=d234e629- } \\
\text { 4509-4e7f-8379-e713efca803c }\end{array}$ \\
\hline
\end{tabular}




\section{Value of the Data}

- The dataset can be used for further analysis of teacher satisfaction and online teaching effectiveness with the focus on the chaotic context of a pandemic.

- The dataset can be used to construct models to evaluate educational leadership and school effectiveness in abnormal situations.

- The significant differences in Vietnamese teachers' income before and during COVID-19 in this dataset can contribute to overall economic models on COVID19's damage.

- The dataset will be useful for school managers and policymakers to renovate policies, regulations, and practices to enhance teacher satisfaction, engagement, and effectiveness.

- The dataset presents a natural flow to measure teacher perceptions and satisfaction during COVID-19, which can be replicated in other countries.

\section{Data Description}

School effectiveness measurements include various factors related to students, teachers, and school managers that affect students' academic achievement [1]. Although the Vietnamese government applied different systematic solutions to minimize the negative impacts of the COVID-19 pandemic [2], there is a lack of empirical evidence to support the decision-making process of school leaders. Under the chaotic circumstances caused by the pandemic, the significant shifts in learning and teaching habits require school leaders to face critical unknown-unknown issues. The formation of this dataset is an extension of our recent study on students' learning habits during the pandemic [3, 4], which contributes to the call of Elsevier on conducting research to tackle the current and potential impairments of the pandemic [5]. Regarding the sudden shift to online teaching and learning due to school closures, this dataset [6] portrayed Vietnamese teachers' perspectives and teaching effectiveness during the pandemic and schools' readiness toward the digital transformation.

Besides the information about the demographics of the participants, this dataset includes two primary groups of research items: (i) Teachers' perceptions of factors associated with online teaching and learning; and (ii) Teachers' opinions on school readiness and teaching effectiveness during the pandemic. The full questionnaires, variable code, and measurement parameters for all research items have been reposited in Harvard Dataverse [6]. Integrations among those variables can examine teacher satisfaction, self-reported teaching effectiveness, and school readiness during the pandemic.

Table 1. Descriptive statistics of participant demographics

\begin{tabular}{|c|c|c|c|c|c|c|c|}
\hline Teacher satisfaction & $\mathrm{N}$ & Mean & $\begin{array}{c}\text { Std. } \\
\text { Deviation }\end{array}$ & $\begin{array}{c}\text { Std. } \\
\text { Error }\end{array}$ & Max & $\begin{array}{c}\text { 95\% Confidence } \\
\text { Interval for Mean }\end{array}$ & Min \\
\hline
\end{tabular}




\begin{tabular}{|c|c|c|c|c|c|c|c|c|c|}
\hline & & & & & & & $\begin{array}{l}\text { Lower } \\
\text { Bound }\end{array}$ & $\begin{array}{l}\text { Upper } \\
\text { Bound }\end{array}$ & \\
\hline \multirow{3}{*}{ Gender } & Male & 46 & 2.772 & 0.828 & 0.122 & 5 & 2.526 & 3.018 & 2 \\
\hline & Female & 245 & 2.929 & 0.776 & $\begin{array}{c}0.05 \\
0\end{array}$ & 5 & 2.831 & 3.026 & 1 \\
\hline & $\begin{array}{l}\text { Prefer not to } \\
\text { disclose }\end{array}$ & 3 & 2.167 & 1.041 & 0.601 & 3 & -0.419 & 4.752 & 1 \\
\hline \multirow{4}{*}{ Exp } & Less than 3 years & 64 & 2.953 & 0.733 & $\begin{array}{c}0.09 \\
2\end{array}$ & 5 & 2.770 & 3.136 & 1 \\
\hline & From 3 to 5 years & 48 & 2.823 & 0.796 & 0.115 & 4 & 2.592 & 3.054 & 1 \\
\hline & $\begin{array}{l}\text { From } 5 \text { to } 10 \\
\text { years }\end{array}$ & 59 & 2.805 & 0.820 & 0.107 & 5 & 2.591 & 3.019 & 1 \\
\hline & $\begin{array}{l}\text { More than } 10 \\
\text { years }\end{array}$ & 123 & 2.939 & 0.804 & $\begin{array}{c}0.07 \\
2 \\
\end{array}$ & 5 & 2.796 & 3.082 & 1 \\
\hline \multirow{4}{*}{ Degree } & Diploma & 13 & 2.615 & 0.506 & 0.140 & 3 & 2.309 & 2.921 & 2 \\
\hline & BA & 181 & 2.909 & 0.759 & $\begin{array}{c}0.05 \\
6 \\
\end{array}$ & 5 & 2.797 & 3.020 & 1 \\
\hline & MA & 89 & 2.888 & 0.878 & $\begin{array}{c}0.09 \\
3 \\
\end{array}$ & 5 & 2.703 & 3.073 & 1 \\
\hline & Doctor & 11 & 3.091 & 0.801 & 0.241 & 4 & 2.553 & 3.629 & 2 \\
\hline \multirow{5}{*}{ Grade level } & Pre-K & 9 & 3.111 & 0.651 & 0.217 & 4 & 2.611 & 3.611 & 2 \\
\hline & Primary & 100 & 2.825 & 0.783 & $\begin{array}{c}0.07 \\
8 \\
\end{array}$ & 5 & 2.670 & 2.980 & 1 \\
\hline & Lower Secondary & 63 & 2.722 & 0.745 & $\begin{array}{c}0.09 \\
4 \\
\end{array}$ & 4 & 2.535 & 2.910 & 1 \\
\hline & Upper Secondary & 66 & 3.068 & 0.784 & $\begin{array}{c}0.09 \\
6\end{array}$ & 5 & 2.875 & 3.261 & 1 \\
\hline & Post-Secondary & 56 & 2.982 & 0.842 & 0.113 & 5 & 2.757 & 3.208 & 1 \\
\hline \multirow{4}{*}{ Subject } & Sciences-related & 87 & 2.948 & 0.743 & $\begin{array}{c}0.08 \\
0\end{array}$ & 5 & 2.790 & 3.107 & 1 \\
\hline & $\begin{array}{l}\text { Social Sciences- } \\
\text { related }\end{array}$ & 70 & 2.971 & 0.751 & $\begin{array}{c}0.09 \\
0\end{array}$ & 5 & 2.792 & 3.151 & 1 \\
\hline & $\begin{array}{l}\text { Foreign } \\
\text { Language }\end{array}$ & 57 & 2.763 & 0.835 & 0.111 & 5 & 2.542 & 2.985 & 1 \\
\hline & Others & 80 & 2.869 & 0.837 & $\begin{array}{c}0.09 \\
4\end{array}$ & 5 & 2.682 & 3.055 & 1 \\
\hline \multirow{5}{*}{ School type } & Public & 191 & 2.901 & 0.747 & $\begin{array}{c}0.05 \\
4\end{array}$ & 5 & 2.794 & 3.007 & 1 \\
\hline & Private (normal) & 49 & 3.041 & 0.822 & 0.117 & 5 & 2.805 & 3.277 & 1 \\
\hline & $\begin{array}{l}\text { Private } \\
\text { (bilingual/intern } \\
\text { ational) }\end{array}$ & 37 & 2.730 & 0.838 & 0.138 & 4 & 2.450 & 3.009 & 1 \\
\hline & $\begin{array}{l}\text { Continuing } \\
\text { Education Center }\end{array}$ & 13 & 2.615 & 0.820 & $\begin{array}{c}0.22 \\
8 \\
\end{array}$ & 4 & 2.120 & 3.111 & 1 \\
\hline & Others & 4 & $3 \cdot 375$ & 1.493 & 0.747 & 5 & 0.999 & $5 \cdot 751$ & 2 \\
\hline \multirow{4}{*}{$\begin{array}{l}\text { Income } \\
\text { before } \\
\text { COVID-19 } \\
\text { pandemic } \\
\text { (USD) }\end{array}$} & $<214$ & 24 & 3.000 & 0.571 & 0.117 & 4 & 2.759 & 3.241 & 2 \\
\hline & $214 \sim 427$ & 124 & 2.927 & 0.823 & $\begin{array}{c}0.07 \\
4 \\
\end{array}$ & 5 & 2.781 & 3.074 & 1 \\
\hline & $427 \sim 641$ & 67 & 2.851 & 0.685 & $\begin{array}{c}0.08 \\
4 \\
\end{array}$ & 5 & 2.684 & 3.018 & 1 \\
\hline & $641 \sim 855$ & 42 & 2.821 & 0.832 & 0.128 & 5 & 2.562 & 3.081 & 1 \\
\hline
\end{tabular}




\begin{tabular}{|c|c|c|c|c|c|c|c|c|c|}
\hline & $>855$ & 37 & 2.892 & 0.936 & 0.154 & 5 & 2.580 & 3.204 & 1 \\
\hline \multirow{5}{*}{$\begin{array}{l}\text { Income } \\
\text { during } \\
\text { COVID-19 } \\
\text { pandemic } \\
\text { (USD) }\end{array}$} & $<214$ & 100 & 2.905 & 0.695 & $\begin{array}{c}0.07 \\
0\end{array}$ & 4 & 2.767 & 3.043 & 1 \\
\hline & $214 \sim 427$ & 133 & 2.868 & 0.790 & $\begin{array}{c}0.06 \\
9\end{array}$ & 5 & 2.733 & 3.004 & 1 \\
\hline & $427 \sim 641$ & 35 & 3.071 & 0.768 & 0.130 & 5 & 2.807 & 3.335 & 2 \\
\hline & $641 \sim 855$ & 19 & 2.684 & 1.157 & 0.265 & 5 & 2.126 & 3.242 & 1 \\
\hline & $>855$ & 7 & 3.000 & 1.000 & 0.378 & 5 & 2.075 & 3.925 & 2 \\
\hline \multirow{5}{*}{$\begin{array}{l}\text { Expected } \\
\text { income } \\
\text { after } \\
\text { COVID-19 } \\
\text { pandemic } \\
\text { (USD) }\end{array}$} & $<214$ & 36 & 2.931 & 0.767 & 0.128 & 4 & 2.671 & 3.190 & 1 \\
\hline & $214 \sim 427$ & 114 & 2.908 & 0.760 & 0.071 & 5 & 2.767 & 3.049 & 1 \\
\hline & $427 \sim 641$ & 84 & 2.881 & 0.767 & $\begin{array}{c}0.08 \\
4\end{array}$ & 5 & 2.715 & 3.047 & 1 \\
\hline & $641 \sim 855$ & 28 & 2.893 & 0.936 & 0.177 & 5 & 2.530 & 3.256 & 1 \\
\hline & $>855$ & 32 & 2.859 & 0.882 & 0.156 & 5 & 2.541 & 3.177 & 1 \\
\hline \multicolumn{2}{|l|}{ Total } & 294 & 2.896 & 0.789 & $\begin{array}{c}0.04 \\
6\end{array}$ & 5 & 2.806 & 2.987 & 1 \\
\hline
\end{tabular}

Table 2. Descriptive statistics of teachers' perceptions of factors that affect their teaching profession during COVID-19 pandemic

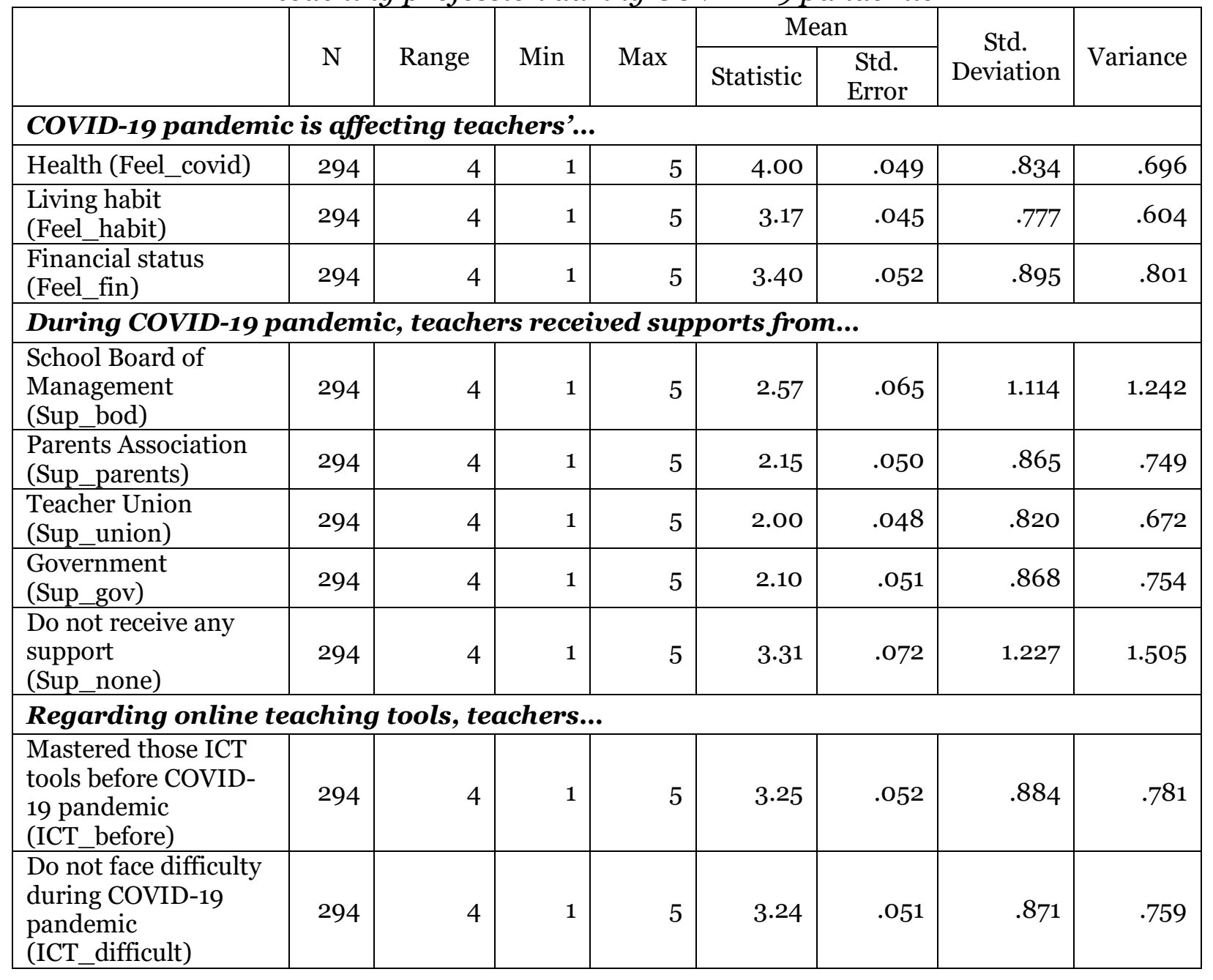




\begin{tabular}{|c|c|c|c|c|c|c|c|c|}
\hline $\begin{array}{l}\text { Know many types of } \\
\text { online teaching tools } \\
\text { (ICT_diverse) }\end{array}$ & 294 & 4 & 1 & 5 & 3.50 & .062 & 1.057 & 1.118 \\
\hline \multicolumn{9}{|c|}{ Teachers often learn new ICT tools... } \\
\hline $\begin{array}{l}\text { Proactively } \\
\text { (ICT_proactive) }\end{array}$ & 294 & 3 & 2 & 5 & 3.62 & .047 & .804 & .647 \\
\hline $\begin{array}{l}\text { More than what } \\
\text { school provides } \\
\text { (ICT_extend) }\end{array}$ & 294 & 3 & 2 & 5 & 3.73 & .045 & .765 & .585 \\
\hline
\end{tabular}

Table 3. Descriptive statistics of teachers' perceptions of school readiness, teaching effectiveness, and professional development during COVID-19 pandemic

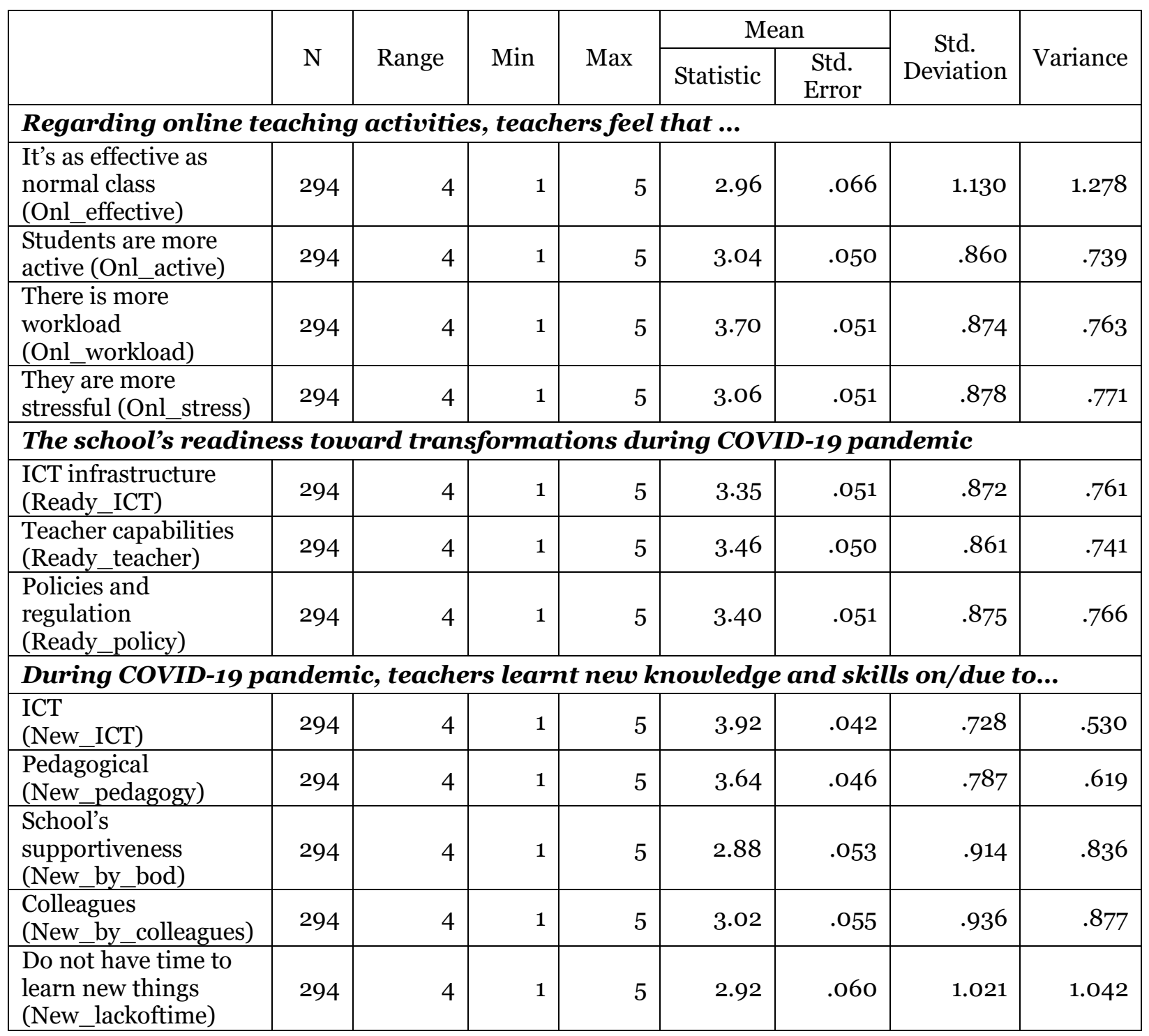

Table 4. Test of Homogeneity of Variances

\begin{tabular}{|c|c|c|c|c|}
\hline & $\begin{array}{c}\text { Levene } \\
\text { Statistic }\end{array}$ & df1 & df2 & Sig. \\
\hline
\end{tabular}




\begin{tabular}{|l|c|c|c|c|} 
Gender & .976 & 2 & 291 & .378 \\
\hline Exp & .887 & 3 & 290 & .448 \\
\hline Degree & 1.424 & 3 & 290 & .236 \\
\hline Grade_level & .282 & 4 & 289 & .889 \\
\hline Subject & .983 & 3 & 290 & .401 \\
\hline School type & 1.416 & 4 & 289 & .229 \\
\hline Income before & 2.102 & 4 & 289 & .081 \\
\hline Income during & 3.183 & 4 & 289 & .014 \\
\hline Income expect & .582 & 4 & 289 & .676 \\
\hline feel covid & .413 & 4 & 289 & .800 \\
\hline Feel habit & .705 & 4 & 289 & .589 \\
\hline feel fin & 1.045 & 4 & 289 & .384 \\
\hline Sup_bod & 2.985 & 4 & 289 & .019 \\
\hline Sup_parents & $3.394 a$ & 3 & 289 & .018 \\
\hline Sup_union & 1.892 & 4 & 289 & .112 \\
\hline Sup_gov & 2.162 & 4 & 289 & .073 \\
\hline Sup_none & 4.093 & 4 & 289 & .003 \\
\hline ICT_before & 2.855 & 4 & 289 & .024 \\
\hline ICT_difficult & .490 & 4 & 289 & .743 \\
\hline ICT_diverse & 2.128 & 4 & 289 & .077 \\
\hline ICT_proactive & 2.565 & 3 & 290 & .055 \\
\hline ICT_extend & 2.732 & 3 & 290 & .044 \\
\hline Onl_effective & 1.333 & 4 & 289 & .258 \\
\hline Onl_active & 5.001 & 4 & 289 & .001 \\
\hline Onl_workload & .730 & 4 & 289 & .572 \\
\hline Onl_stress & 1.384 & 4 & 289 & .239 \\
\hline Ready_ICT & 4.785 & 4 & 289 & .001 \\
\hline Ready_teacher & 4.552 & 4 & 289 & .001 \\
\hline Ready_policy & 3.714 & 4 & 289 & .006 \\
\hline New_ICT & 2.163 & 4 & 289 & .073 \\
\hline New_pedagogy & .214 & 4 & 289 & .930 \\
\hline New_by_bod & 7.690 & 4 & 289 & .000 \\
\hline New_by_colleagues & 9.253 & 4 & 289 & .000 \\
\hline New_lackoftime & 3.597 & 4 & 289 & .007 \\
\hline & & & & \\
\hline
\end{tabular}

Table 5. Differences in teachers' satisfaction during COVID-19 pandemic among different demographics (ANOVA analysis)

\begin{tabular}{|l|l|r|r|r|r|r|}
\hline \multicolumn{2}{|c|}{ Tearcher satisfaction } & $\begin{array}{c}\text { Sum of } \\
\text { Squares }\end{array}$ & \multicolumn{1}{c|}{ df } & $\begin{array}{c}\text { Mean } \\
\text { Square }\end{array}$ & \multicolumn{1}{c|}{ F } & \multicolumn{1}{c|}{ Sig. } \\
\hline \multirow{2}{*}{ Gender } & Between Groups & 2.566 & 2 & 1.283 & 2.074 & .128 \\
\cline { 2 - 8 } & Within Groups & 180.020 & 291 & .619 & & \\
\hline \multirow{2}{*}{ Exp } & Between Groups & 1.181 & 3 & .394 & .629 & .597 \\
\cline { 2 - 8 } & Within Groups & 181.405 & 290 & .626 & & .501 \\
\hline \multirow{2}{*}{ Degree } & Between Groups & 1.478 & 3 & .493 & .789 & .501 \\
\cline { 2 - 8 } & Within Groups & 181.108 & 290 & .625 & & .079 \\
\hline Grade level & Between Groups & 5.195 & 4 & 1.299 & 2.116 & \\
\hline
\end{tabular}




\begin{tabular}{|c|c|c|c|c|c|c|}
\hline & Within Groups & 177.391 & 289 & .614 & & \\
\hline & Total & 182.586 & 293 & & & \\
\hline \multirow{2}{*}{ Subject } & Between Groups & 1.701 & 3 & .567 & .909 & .437 \\
\hline & Within Groups & 180.885 & 290 & .624 & & \\
\hline \multirow{2}{*}{ School type } & Between Groups & 3.996 & 4 & .999 & 1.617 & .170 \\
\hline & Within Groups & 178.590 & 289 & .618 & & \\
\hline \multirow{2}{*}{ Income before } & Between Groups & .753 & 4 & .188 & .299 & .878 \\
\hline & Within Groups & 181.833 & 289 & .629 & & \\
\hline \multirow{2}{*}{ Income expect } & Between Groups & .121 & 4 & .030 & .048 & .996 \\
\hline & Within Groups & 182.465 & 289 & .631 & & \\
\hline \multicolumn{2}{|c|}{ Total } & 182.586 & 293 & & & \\
\hline
\end{tabular}

Table 6. Differences in teachers' satisfaction during COVID-19 pandemic among different examined perspectives (ANOVA analysis)

\begin{tabular}{|c|c|c|c|c|c|c|}
\hline \multicolumn{2}{|c|}{ Tearcher satisfaction } & $\begin{array}{l}\text { Sum of } \\
\text { Squares }\end{array}$ & $\mathrm{df}$ & $\begin{array}{l}\text { Mean } \\
\text { Square }\end{array}$ & $\mathrm{F}$ & Sig. \\
\hline \multirow{2}{*}{ Feel_covid } & $\begin{array}{l}\text { Between } \\
\text { Groups }\end{array}$ & $7 \cdot 589$ & 4 & 1.897 & 3.133 & .015 \\
\hline & Within Groups & 174.997 & 289 & .606 & & \\
\hline \multirow{2}{*}{ Feel_habit } & $\begin{array}{l}\text { Between } \\
\text { Groups } \\
\end{array}$ & 18.811 & 4 & 4.703 & 8.299 & .000 \\
\hline & Within Groups & 163.775 & 289 & .567 & & \\
\hline \multirow{2}{*}{ Feel_fin } & $\begin{array}{l}\text { Between } \\
\text { Groups }\end{array}$ & 11.009 & 4 & 2.752 & 4.636 & .001 \\
\hline & Within Groups & 171.577 & 289 & .594 & & \\
\hline \multirow{2}{*}{ Sup_union } & $\begin{array}{l}\text { Between } \\
\text { Groups }\end{array}$ & 26.275 & 4 & 6.569 & 12.145 & .000 \\
\hline & Within Groups & 156.310 & 289 & .541 & & \\
\hline \multirow{2}{*}{ Sup_gov } & $\begin{array}{l}\text { Between } \\
\text { Groups }\end{array}$ & 25.849 & 4 & 6.462 & 11.915 & .000 \\
\hline & Within Groups & 156.737 & 289 & .542 & & \\
\hline \multirow{2}{*}{ ICT_difficult } & $\begin{array}{l}\text { Between } \\
\text { Groups }\end{array}$ & $3 \cdot 788$ & 4 & .947 & 1.531 & .193 \\
\hline & Within Groups & 178.798 & 289 & .619 & & \\
\hline \multirow{2}{*}{ ICT_diverse } & $\begin{array}{l}\text { Between } \\
\text { Groups }\end{array}$ & 2.524 & 4 & .631 & 1.013 & .401 \\
\hline & Within Groups & 180.062 & 289 & .623 & & \\
\hline \multirow{2}{*}{ ICT_proactive } & $\begin{array}{l}\text { Between } \\
\text { Groups } \\
\end{array}$ & 1.443 & 3 & .481 & .770 & .512 \\
\hline & Within Groups & 181.143 & 290 & .625 & & \\
\hline \multirow[t]{2}{*}{ Onl_effective } & $\begin{array}{l}\text { Between } \\
\text { Groups }\end{array}$ & 5.463 & 4 & 1.366 & 2.228 & .066 \\
\hline & Within Groups & 177.123 & 289 & .613 & & \\
\hline \multirow{2}{*}{ Onl_workload } & $\begin{array}{l}\text { Between } \\
\text { Groups }\end{array}$ & 2.107 & 4 & .527 & .844 & .498 \\
\hline & Within Groups & 180.478 & 289 & .624 & & \\
\hline
\end{tabular}




\begin{tabular}{|l|l|r|r|r|r|r|}
\hline \multirow{2}{*}{ Onl_stress } & $\begin{array}{l}\text { Between } \\
\text { Groups }\end{array}$ & 5.803 & 4 & 1.451 & 2.372 & .053 \\
\cline { 2 - 7 } & Within Groups & 176.783 & 289 & .612 & & \\
\hline \multirow{2}{*}{ New_ICT } & $\begin{array}{l}\text { Between } \\
\text { Groups }\end{array}$ & 7.422 & 4 & 1.856 & 3.061 & .017 \\
\cline { 2 - 7 } & Within Groups & 175.164 & 289 & .606 & & \\
\hline \multirow{2}{*}{ New_pedagogy } & $\begin{array}{l}\text { Between } \\
\text { Groups }\end{array}$ & 8.382 & 4 & 2.095 & 3.476 & .009 \\
\cline { 2 - 7 } & Within Groups & 174.204 & 289 & .603 & & \\
\hline \multicolumn{2}{|r|}{ Total } & 182.586 & 293 & & & \\
\hline
\end{tabular}

Table 7. Robust Tests of Equality of Means toward Teacher Satisfaction

\begin{tabular}{|l|r|r|r|r|r|}
\hline & Welch & \multicolumn{1}{c|}{ Statistic* } & \multicolumn{1}{c|}{ df1 } & \multicolumn{1}{c|}{ df2 } & \multicolumn{1}{c|}{ Sig. } \\
\hline Income_during & & .632 & 4 & 32.880 & .643 \\
\hline Sup_bod & & 5.665 & 4 & 66.141 & .001 \\
\hline Sup_parents** & & & & & \\
\hline Sup_none & & 7.515 & 4 & 85.241 & .000 \\
\hline ICT_before & & .911 & 4 & 25.462 & .473 \\
\hline ICT_extend & & 2.066 & 3 & 53.860 & .116 \\
\hline Onl_active & & 1.466 & 4 & 38.295 & .231 \\
\hline Ready_ICT & & 6.891 & 4 & 25.282 & .001 \\
\hline Ready_teacher & & 6.968 & 4 & 19.962 & .001 \\
\hline Ready_policy & & 7.612 & 4 & 30.007 & .000 \\
\hline New_by_bod & & 9.912 & 4 & 32.264 & .000 \\
\hline New_by_colleagues & & 1.146 & 4 & 29.849 & .354 \\
\hline New_lackoftime & & 5.489 & 4 & 56.265 & .001 \\
\hline
\end{tabular}

* Asymptotically F distributed.

** Robust tests of equality of means cannot be performed for Tearcher satisfaction because at least one group has the sum of case weights less than or equal to 1.

\section{Experimental Design, Materials, and Methods}

The data was collected from 6th to 11th April 2020, the ninth week of national school suspension in Vietnam, due to the COVID-19 pandemic. Considering that there are more than one million teachers in Vietnam, it is impossible to reach all types of teachers across the country. Thus, the researchers focused on the two biggest teacher communities on Facebook: Microsoft Innovative Education Expert Vietnam - MIE (38,600 members) and Vietnam Innovative Education Forum - VIEF (14,000 members). Firstly, the survey was announced by the admins of those groups and attracted around 500 interactions from members. Additionally, we randomly selected 1,000 members from each group and sent them the survey URL, separately. Overall, a total of 373 responses was collected. Couples of cross-checking questions with reversed Linkert scales were embedded in the survey and helped us to eliminate 79 bias observations. Finally, we analyzed the dataset of 294 respondents. 
The differences between teachers' satisfaction among various demographic indicators and examined research items can be presented through ANOVA analysis. In particular, Table 4 shows the test of homogeneity of variances. Table 5 and Table 6 display the differences in teachers' satisfaction among demographic indicators and teachers' perception, respectively. The results of robust tests of equality of means are included in Table 7.

Using questions with the five-points Linkert scale, this dataset demonstrated the factors associated with online teaching effectiveness, teacher satisfaction, and school effectiveness during the pandemic.

Regarding the control over online teaching effectiveness (ONL_EFF), we considered four factors. First, teachers' overall perceptions of the impact of the pandemic (FEEL) are the aggregated result of the influence of the pandemic on their health; their living habits; and their financial status $[7,8]$. Second, we indicated the teachers' received support (SUP) as a function of the support they receive from: School Board of Management; Parents Association; Teacher union; and Government bodies [9, 10]. The question "I do not receive any support" was included to cross-check the validity of respondents. Third, teachers' capability toward online teaching technologies (ICT_CAP) was the mean of their self-reported ICT (Information and Communication Technology) competency [10] before the pandemic emerged; the smooth of their online lesson during the pandemic; and the diversity of the tools which they mastered. Also, we added additional questions to examine the teacher's proactiveness in learning new ICT tools (ICT_ACT). We consider the influence of the above factors over online teaching effectiveness by the following regression:

$$
\text { ONL_EFF } \sim \beta \mathrm{O}+\beta 1^{*} \mathrm{FEEL}+\beta 2^{*}(\mathrm{SUP})+\beta 3^{*}\left(\mathrm{ICT} \_\mathrm{CAP}\right)+\beta 4^{*}\left(\mathrm{ICT} \_\mathrm{ACT}\right)+\mathrm{u}
$$

Regarding the influence over teacher satisfaction, we included teachers' self-reports among the three following constructs [11]. First, teachers' perceptions of online teaching activities (ONL_PER) were combined from the effectiveness of online class (in comparison with regular lessons - Onl_effective) [12], students' activeness (Onl_active) [13], workload increment (Onl_workload), and level of stress during the pandemic (Onl_stress) [14]. During further analytical processes, the measurement scale of increased workload and degree of stress should be reversed to ensure the consistency of the overall construct. Second, the school's readiness toward digital transformations during the pandemic (READY) was indicated by the eagerness of ICT infrastructure, teacher capabilities, policies, and regulation [15]. Third, regarding professional development, we included types and sources of new know-how that teachers absorbed during the pandemic (PD). A cross-checking question was added to exclude invalid answers "I do not have time to learn new things." If the response of this question is not consistent with the previous three, we will eliminate that observation. Considering 
teacher satisfaction as the primary outcome, the influence of those other factors listed above can be examined by the following regression:

$$
\mathrm{SAT} \sim \beta \mathrm{O}+\beta 1^{*}\left(\mathrm{ONL} \_\mathrm{PER}\right)+\beta 2^{*}(\mathrm{READY})+\beta 3^{*}(\mathrm{PD})+\mathrm{u}
$$

\section{Acknowledgments}

A great thanks to all the teachers who participated in this study, as well as the community managers of Microsoft Innovative Education Forum Vietnam (MIE Vietnam) and Vietnam Innovative Education Forum (VIEF), school leaders, and instructional coaches who contributed to elevating the data collection process.

\section{Competing Interests}

The authors declare that they have no known competing financial interests or personal relationships which have, or could be perceived to have, influenced the work reported in this article.

\section{References}

[1] Gamoran, A., \& Long, D. A. (2007). Equality of educational opportunity a 40 year retrospective. In International studies in educational inequality, theory and policy (pp. 23-47). Springer, Dordrecht.

[2] La, V., Pham, T., Ho, T. M., Hoàng, N. M., Linh, N. P. K., Vuong, T., ... Vuong, Q. (2020). Policy response, social media and science journalism for the sustainability of the public health system amid COVID-19 outbreak: The Vietnam lessons. Sustainability, 12, 2931. https://doi.org/10.3390/su12072931

[3] Tran, T., Hoang, A. D., Nguyen, T. T., Dinh, V.H., Nguyen, Y. C., Pham, H. H. (2020). Dataset of Vietnamese student's learning habits during COVID-19. Data in Brief, $105682 \mathrm{https}$ ://doi.org/10.1016/j.dib.2020.105682

[4] Tran, T., Hoang, A. D., Nguyen, Y. C., Nguyen, L. C., Ta, N. T., Pham, Q. H., Pham, C. X., Le, Q. A., Dinh, V. H., Nguyen, T. T. (2020). Toward Sustainable Learning during School Suspension: Socioeconomic, Occupational Aspirations, and Learning Behavior of Vietnamese Students during COVID-19. Sustainability, 12(10):4195. https://doi.org/10.3390/su12104195

[5] Elsevier (2020). Novel Coronavirus Information Center. Elsevier. https://www.elsevier.com/connect/coronavirus-information-center [6] [dataset] Hoang, Anh-Duc; Hiep-Hung Pham; Quynh-Anh Thi Le; Viet-Hung Dinh; Thu-Trang Thi Le; Yen-Chi Nguyen, 2020, "Survey on Vietnamese teachers' perspectives during COVID-19", https://doi.org/10.7910/DVN/FOCPKH, Harvard Dataverse, V1 [7] Hsu, H. I., \& Tsai, H. M. (2013). A study of social support, self-efficacy and school effectiveness of teachers in New Taipei City, Chung Yuan Physical Education Journal, Vol. 3, 68-75.

[8] Barr, A. (1952). The measurement of teacher characteristics and prediction of teaching efficiency. Review of Educational Research, 22, 169-174. [9] Holt-Lunstad J, Smith TB, Layton JB (2010) Social Relationships and Mortality Risk: A Meta-analytic Review. PLoS Med 7(7): e1000316. 
[10] Toker, B. (2011), "Job satisfaction of academic staff: an empirical study on Turkey", Quality Assurance in Education, Vol. 19 (2), pp. 156-169.

[11] Hernández-Ramos, J. P., Martínez-Abad, F., Peñalvo, F. J. G., García, M. E. H., \& Rodríguez-Conde, M. J. (2014). Teachers' attitude regarding the use of ICT. A factor reliability and validity study. Computers in Human Behavior, 31, 509-516.

[12] Dinham, S. and Scott, C. (1998), "A three domain model of teacher and school executive satisfaction", Journal of Educational Administration, Vol. 36 No. 4, pp. 362-78. [13] Day, C. (2013). The new lives of teachers. In Back to the Future (pp. 57-74). Brill Sense.

[14] Dörnyei, Z., \& Ushioda, E. (2013). Teaching and researching: Motivation.

Routledge.

[15] Sahito, Z., \& Vaisanen, P. (2017). Effect of ICT Skills on the Job Satisfaction of Teacher Educators: Evidence from the Universities of the Sindh Province of Pakistan. International journal of higher education, 6(4), 122-136.s 